\title{
Calidad de servicio y valor percibido como antecedentes de la satisfacción de los clientes de las empresas de telecomunicaciones en Colombia
}

\author{
Service quality and perceived value as users' satisfaction antecedents of \\ Colombia's telecommunications enterprises
}

\section{Laura Cristina Henao Colorado}

Instituto Tecnológico Metropolitano, Colombia

Recibido el 28 de diciembre de 2018; aceptado el 13 de mayo de 2019

Disponible en Internet el: 21 de mayo de 2019

\section{Resumen}

A pesar de las diferentes críticas que ha recibido el modelo SERVQUAL propuesto por Zeithaml, Parasuraman y Berry, este aún sigue vigente y ha permitido mostrar que la calidad de servicio es antecedente de la satisfacción de los clientes. Para el sector de las telecomunicaciones en Colombia, donde las empresas son pocas, pero con una alta imitación de ofertas, el entender si sus clientes están satisfechos, cómo perciben los servicios y qué valoran más de ellos, se ha vuelto en factores de competitividad. Es así como el presente estudio, propone un modelo que explica cómo influyen las expectativas (EXP), la calidad de servicio (QS), y el valor percibido (VP) en la satisfacción (SAT) de los clientes de las empresas de telecomunicaciones fijas en Colombia. Se obtuvieron datos de 324 usuarios en las principales ciudades del país, con un cuestionario virtual. Las 5 hipótesis sugeridas se probaron utilizando modelos de ecuaciones estructurales por PLS-SEM. Los hallazgos evidencian la pertinencia del SERVQUAL para explicar la calidad de servicio y que la satisfacción está influenciada directamente por dicho constructo

\footnotetext{
*Autor para correspondencia

Correo electrónico laurahenao@itm.edu.co (L.C. Henao Colorado).

La revisión por pares es responsabilidad de la Universidad Nacional Autónoma de México. 
y el valor percibido. Esta investigación es una contribución de la literatura dada la poca investigación sobre estos constructos en dicho sector y país.

Código JEL: M00, M30, M31

Palabras clave: SERVQUAL; Satisfacción; Valor percibido; Marketing; Telecomunicaciones

\begin{abstract}
Despite the different criticisms received by the Zeithaml, Parasuraman and Berry model, the SERVQUAL is valid and has shown that the service quality is a satisfaction's customer precedent. To the Colombia's telecommunications sector, where are few companies with a high imitation offers, to understand if their customers are satisfied, their service perceptions and the value their clients perceive, has become competitive factors. The present study proposes a model that explains how expectations (EXP), service quality (QS), and perceived value (VP) affect the satisfaction (SAT) construct of Colombia's fixed telecommunications companies. Empirical data was obtained from 324 users in the country's main cities by virtual questionnaire. The 5 suggested hypotheses were tested using structural equation models by PLS-SEM. The findings show the relevance of SERVQUAL to explain the service quality construct and its relationship with the satisfaction. Given the limited research on these constructs in this sector, this research is a contribution to the literature.
\end{abstract}

JEL codes: M00, M30, M31

Keywords: SERVQUAL; Satisfaction; Perceived value; Marketing; Telecommunications

\title{
Introducción
}

Las empresas hoy, deben concentrar sus esfuerzos y direccionar sus recursos en el conocimiento del cliente; es decir, en saber qué esperan y cómo perciben los servicios ofertados (Tsafaraski, Kokotas \& Pantouvakis, 2018). Basados en lo anterior, estas empresas deben pasar de una lógica de gestión de bienes a una de servicios, por medio de la implementación de estrategias enfocadas en la satisfacción de los clientes (Zeithaml, Bitner \& Gremler, 2009), transcendiendo así a un marketing relacional. Para lo anterior, factores como la calidad de servicio y el valor percibido, deben ser tenidos en cuenta y con esto lograr diferenciarse de la competencia (Tsafaraski et al., 2018).

Para el sector de las telecomunicaciones, esta lógica enfocada en el cliente, no es diferente, y más en un país como Colombia, donde el sector cuenta con un número de competidores que va en ascenso (Algarra, 2018), que incurren en costos fijos altos y se caracterizan por su alta imitación de ofertas. Además, se viene dando un incremento en el uso de los servicios con buenos resultados (Combariza, García, Alvarado, España \& Rivera, 2012). Adicional 
a ello, el sector es muy dinámico, con una demanda de conexiones de banda ancha fija en aumento y con creaciones de planes cada vez más adaptados a las necesidades de los clientes (Combariza et al., 2012).

A pesar de dicho dinamismo, se viene presentando una disminución en la demanda de servicios fijos domiciliarios desde 2011, dada la portación a servicios móviles (MINTIC, 2016) reflejada en 35 millones de colombianos portados de un operador a otro para 2015 (El Tiempo, 2015). Por lo tanto, las empresas deben procurar satisfacer a sus clientes, para que cambien de tipo de servicio, pero no de operador, y esto lo pueden lograr brindando una buena calidad de servicio y tratando de superar las expectativas de los clientes (Zeithaml et al., 1991). Además, deben revisar otros antecedentes de la satisfacción, como el valor percibido (Lam, Shankar, Erramilli \& Murthy, 2004), al ser considerado un factor más completo de valoración del servicio (Bolton \& Drew, 1991).

Dado lo anterior, este estudio tiene como objetivo proponer un modelo conceptual que muestre los antecedentes de la satisfacción de los clientes de las empresas de telecomunicaciones de las principales ciudades de Colombia, como lo son la calidad de servicio, adaptando el modelo SERVQUAL, y el valor percibido. Es así como, en la primera parte, se presenta la revisión de la literatura, donde se destacan sectores como el de la banca, salud, educación y turismo (López \& Serrano, 2001; Naik, Gantasala \& Prabhakar, 2010; Yépez, Ricaurte \& Jurado, 2018); y el sector de telecomunicaciones, con estudios a nivel internacional (Boohene \& Agyapong, 2011; Arslan, Iftikhar \& Zaman, 2014; Bustamante, 2015), aportando, por tanto, a los estudios en este campo, en un sector de Colombia donde no se identificaron investigaciones de este tipo. En la segunda parte, se detalla la sección de la metodología. Luego, se discuten los resultados, y se finaliza con las conclusiones e implicaciones de investigación futura.

\section{Marco teórico}

\section{Expectativas}

Las expectativas, en un mercado competitivo y con muchos oferentes, se forman por parte de los consumidores de acuerdo con las alternativas que se les presenta (Naumann, Williams \& Khan, 2009). Este constructo es definido como un marco de referencia para predecir qué pasará en una nueva interacción con la empresa y con éste se forman niveles de servicio ideales, deseados o adecuados (Churchill Jr. \& Surprenant, 1982; Berry, Zeithaml \& Parasurama, 1991; 1993; Zeithaml, Bitner \& Gremler, 2009; Vigg \& Bhargav, 2014).

La importancia de este constructo se evidencia en el impacto que tiene en las evaluaciones que hace el cliente luego del uso del servicio (Nath, Devlin \& Reid, 2018). Lo anterior, se ha sugerido en estudios donde se plantean que las expectativas sirven para implementar estrategias 
que provean un servicio superior (Parasuraman et al., 1991; 1993), y se tiene algo similar para el sector de las telecomunicaciones, donde se sugiere los oferentes se centren en actividades que ayuden a cumplir o superar las expectativas de sus clientes (Vigg \& Bhargav, 2014).

\section{Calidad de Servicio}

La evaluación de la calidad de servicio nace de la opinión del cliente quien informa a la empresa si está ofreciendo un servicio con una calidad alta o baja (Strenitzerová \& Gaňa, 2018). Este factor es por tanto entendido como la comparación entre las expectativas y la experiencia que tiene el cliente con el rendimiento del servicio (Parasuraman, et al., 1985; Grönroos, 1984; Berry et al., 1985; Bolton \& Drew, 1991; Boohene \& Agyapong, 2011; Vigg \& Bhargav, 2014); y, cuando se trata de evaluar dicha calidad, se dan respuestas cognitivas a nivel de atributos del servicio como lo son los aspectos tangibles y el aspecto interactivo proporcionado desde los empleados (Chiou \& Droge, 2006).

Para esta investigación, se acepta la definición de Parasuraman et al. (1985; 1993), dada su aceptación en muchos sectores económicos, y por contener factores como las expectativas y la percepción del servicio. Además, su definición tiene referentes adaptables al contexto de las telecomunicaciones, como lo son las 5 dimensiones determinantes de la calidad de servicio. Por tanto, la calidad de servicio se define como el juicio global del cliente sobre la superioridad del servicio, que resulta de comparar sus expectativas y percepciones sobre el servicio ofrecido.

A continuación, en la Tabla 1, se relacionan las 5 dimensiones determinantes de la calidad de servicio.

Tabla 1

Dimensiones SERVQUAL

\begin{tabular}{|c|c|}
\hline Dimensión & Definición \\
\hline Confiabilidad & Habilidad para ofrecer un servicio confiable y consecuente. \\
\hline Capacidad de respuesta & Disposición para brindar un servicio ágil y ayudando a los usuarios. \\
\hline Seguridad & $\begin{array}{l}\text { Preparación de los empleados, cortesía, y habilidad para transmitir confianza y se- } \\
\text { guridad. }\end{array}$ \\
\hline Empatía & Proveer cuidado y atención individual y personalizada a los clientes. \\
\hline Elementos Tangibles & $\begin{array}{l}\text { Apariencia de las instalaciones físicas, equipo, personal y materiales para comuni- } \\
\text { caciones. }\end{array}$ \\
\hline
\end{tabular}

Fuente: Parasuraman et al. (1985; 1993) 
Muchos estudios han evaluado la calidad de servicio con el SERVQUAL. En Venezuela para el sector de las telecomunicaciones y, en Colombia, para el sector hotelero, salud y educación, se tiene evidencia de que la mejor forma de que los clientes elijan una empresa es mejorando la calidad de sus servicios (López \& Serrano, 2001; Wang, Lo \& Yang, 2004; Valdunciel, Flórez \& Dávila, 2007; Losada \& Rodriguez, 2007; Pineda, Estrada \& Parra, 2011; Boohene \& Agyapong, 2011; Vergara, Quesada \& Blanco, 2011; Galbán, Clemenza \& Araujo, 2013; Bustamante, 2015; Meesala \& Paul, 2018; Yépez et al., 2018). Para las telecomunicaciones en Colombia, no se han hecho adaptaciones del SERVQUAL.

\section{Relación Expectativas y Calidad de Servicio}

Algunos estudios han demostrado que un factor determinante de la calidad de servicio en general es la brecha que se da entre el rendimiento del servicio y lo que se esperaba de éste (Bolton \& Drew, 1991; Parasuraman et al., 1991; 1993), siendo por tanto el constructo expectativas un antecedente de la calidad de servicio y con un efecto positivo a través de la percepción del rendimiento/funcionamiento (Spreng \& Mackoy, 1996).

\section{Elección del modelo SERVQUAL para evaluar la calidad de servicio}

El SERVQUAL es un modelo que ha recibido críticas de algunos autores, y entre ellos están Cronin \& Taylor (1992; 1994), quienes proponen el modelo SERVPERF, sin la inclusión de las expectativas; sin embargo, se logra identificar que, a pesar de la omisión de dicho factor en el modelo, sugieren la existencia del constructo al reconocer su diferencia frente a la satisfacción. Además, aunque se ha querido demostrar la superioridad de un modelo sobre el otro, tanto SERVPERF como SERVQUAL, tienen un poder predictivo similar (Miranda, Tavares \& Queiró, 2018), y existe evidencia de que la implementación del SERVQUAL es posible en múltiples contextos en todo el mundo (por ejemplo, Vigg \& Bhargav, 2014; Strenitzerová \& Gaňa, 2018), sin implicar inestabilidad en las dimensiones.

Soportando lo anterior, existen modelos para revisar la calidad de servicio y su relación con factores como las expectativas y la satisfacción, y muchos de estos con adaptaciones del modelo SERVQUAL a las condiciones del sector específico a evaluar. Por un lado, existe el modelo MUSA (Tsafaraski et al., 2018); también se tiene a Roca, Cabello, González \& Courel (2018), con el modelo EVENTQUAL, para mega-eventos deportivos. Y, por último, se tiene a Miranda et al. (2018), con una extensión del modelo SERVQUAL para la industria ferroviaria. Por tanto, se acepta el modelo SERVQUAL para adaptar las dimensiones al sector de las telecomunicaciones en Colombia, y se establece la siguiente hipótesis:

H1: Las expectativas tienen una influencia positiva y directa sobre la calidad de servicio que ofrecen las empresas de telecomunicaciones. 


\section{Satisfacción}

Umar \& Bahrun (2017), definen la satisfacción como la evaluación posterior a la comparación entre las expectativas y la experiencia de compra por parte del cliente. Este constructo es concebido como la actitud frente a la suma de las satisfacciones con los atributos presentes en un servicio (Churchill Jr. \& Surprenant, 1982; Bolton \& Drew, 1991; Carrizo-Moreira, Freitas-Da Silva \& Ferreira-Moutinho, 2017), y es un importante factor en la recompra de productos, específicamente cuando hay intangibilidad y se convierte en un elemento que aumenta cuando el rendimiento del servicio supera las expectativas (Meesala \& Paul, 2018).

Es así como la satisfacción se da desde el placer que produce el consumo, al logra cubrir una necesidad y generar con ello una sensación o sentimiento agradable comparado con un patrón de placer o displacer (Oliver, 1999a; Kim, Park \& Jeong, 2004). Además, la disconformidad (expectativas frente a la experiencia) muestra si la satisfacción es alta o baja cuando las expectativas se superan o caen por debajo de lo que el cliente esperaba (Churchill Jr. \& Surprenant, 1982; Bolton \& Drew, 1991; Boohene \& Agyapong, 2011).

\section{Relación entre expectativas, calidad de servicio y satisfacción}

A nivel mundial se ha comprobado la existencia de relaciones entre las expectativas, la calidad de servicio y la satisfacción (Oliver, 1980; Churchill \& Surprenant, 1982; Parasuraman et al. 1985; 1993; Bolton \& Drew, 1991; Spreng \& Mackoy, 1996; Arslan et al., 2014; Miranda et al., 2018; Vijayakumar \& Shivdas, 2018). En Latinoamérica, también se han demostrado estas relaciones con trabajos como el de Bustamante (2015), quien sugiere que, si se provee un mal servicio, se crea una experiencia insatisfactoria en lo clientes, siendo necesario ofrecer un conjunto consistente de experiencias satisfactorias para obtener una evaluación positiva de la calidad de servicio (Galbán et al., 2013). Por lo tanto, las dimensiones de calidad deben ser usadas para lograr satisfacer a los clientes (Tsafaraski et al., 2018).

Por otro lado, cuando se trata de la utilidad que genera el servicio, la calidad técnica es la que produce satisfacción; y cuando es algo emocional, la satisfacción se da desde la calidad en la interacción con el personal del servicio (Chiou \& Droge, 2006; Kasiri, Cheng, Sambasivanc, \& Sidin, 2017). Para el sector de las telecomunicaciones, se han demostrado dichas relaciones, con la adaptación del modelo SERVQUAL, y donde se destacan aspectos que van desde la calidad en la red, calidad de la llamada, la estructura de precios, hasta la capacidad de respuesta de los empleados (Wang et al., 2004; Park \& Jeong, 2004; Boohene \& Agyapong, 2011; Rahhal, 2015). A nivel Colombia, no se tienen estudios específicos sobre estas relaciones en el sector de las telecomunicaciones, pero sí para el sector bancario (Valdunciel et al., 2007). 
Dado lo anterior, se establecen las siguientes hipótesis:

H2: las expectativas influyen de manera directa y positiva sobre la satisfacción del cliente de las empresas de telecomunicaciones

H3: la calidad de servicio influye de manera directa y positiva sobre la satisfacción del cliente de las empresas de telecomunicaciones.

\section{Valor Percibido}

El valor percibido es una evaluación donde se confrontan los beneficios y sacrificios en los que incurren un cliente con el uso del servicio, y que contiene tanto una dimension afectiva (Escamilla \& Núñez, 2014), como una cognitiva (Lam et al., 2004). Esto, porque los sacrificios o costos se refieren tanto a los monetarios como no monetarios (esfuerzo, tiempo) y se comparan con un marco de referencia que el cliente tenga (Zeithaml, 1988; Bolton \& Drew 1991; Ravald \& Grönroos, 1996). Por otro lado, Oliver (1999b), sugiere basado en los modelos de Zeithaml, que el valor es una función positiva de lo que se recibe y una función negativa de lo que se sacrifica.

Es por tanto, el valor, una evaluación que plantea varias facetas; por un lado, desde lo relativo, por el involucramiento de las preferencias de los clientes que se convierten en elementos para dar un juicio a través de los servicios; y por otro lado, desde la experiencia, porque el valor no solo depende de la compra en sí, sino de las vivencias derivadas del consumo (Holbrook, 1999). Adicional, Konuk (2018), sugiere que la utilidad y los costos son componentes importantes de ese valor percibido, y un precio bajo puede también incrementar ese valor que percibe el cliente.

\section{Relación entre calidad de servicio, satisfacción y valor percibido}

Las empresas pueden proponerle valor al cliente tratando de mejorar la satisfacción de éste, y considerando en sus estrategias no solo lo que se les ofrece, sino también lo que dicho cliente tiene que sacrificar para acceder al producto o servicio (Zeithaml, 1988; Bolton \& Drew 1991; Ravald \& Grönroos, 1996; Taylor \& Hunter, 2003). Estudios a nivel mundial han demostrado la existencia de la relación entre calidad de servicio, satisfacción y valor percibido (por ejemplo, Konuk, 2018; Vijayakumar \& Shivdas, 2018). En Colombia, no se tienen estudios que evidencien la relación entre estos constructos en el sector de las telecomunicaciones. Solo se evidencian estudios para el país, para el sector salud y educación (Vergara \& Quesada, 2011; Betancur, Montoya \& Tavera, 2017).

A pesar de lo anterior, investigaciones internacionales, para dicho sector, sí han logrado demostrar la relación directa entre la calidad de servicio y el valor percibido. El constructo 
satisfacción, también ingresa en la relación con la calidad de servicio, pero mediada por el valor percibido. Esto se argumenta en el hecho de que los clientes no se van a sentir satisfechos con una oferta de calidad, si lo que valoran es otro atributo del servicio que no se incluye en dicha calidad. Por lo tanto, el impacto en la satisfacción va a depender no solo de un servicio de calidad, sino que, este constructo va a valer la pena si los clientes ven en ello un valor que se está mejorando (Wang et al., 2004; Bustamante, 2015).

Dado lo anterior, se establecen las siguientes hipótesis:

H4: la calidad de servicio influye de manera positiva y directa sobre el valor percibido del cliente de las empresas de telecomunicaciones.

H5: el valor percibido del cliente influye de manera positiva y directa sobre la "satisfacción" del cliente de las empresas de telecomunicaciones.

\section{Metodología}

\section{Variables de medida}

Para la medida de los constructos expectativas y calidad de servicio, se adaptó el modelo SERVQUAL de Parasuraman et al. (1985; 1993), y se usaron 22 ítems correspondientes a la percepción y 5 ítems por dimensión para evaluar las expectativas. Se usaron 5 ítems para el constructo satisfacción (Taylor \& Hunter, 2003; Wang et al., 2004; Kim et al., 2004; Chiou \& Droge, 2006; Bustamante, 2015). Y, por último, 5 ítems para el factor valor percibido (Sirdeshmukh et al., 2002; Wang et al., 2004). Las variables fueron adaptadas al contexto de las telecomunicaciones y se midieron utilizando una escala Likert de 5 puntos que varió entre $1=$ muy en desacuerdo y $5=$ muy de acuerdo, para todos los constructos.

Por otro lado, la validez de contenido para las escalas de medida, se llevó a cabo con una encuesta piloto aplicada a expertos en los constructos estudiados. Dichos expertos pertenecen a la Universidad de Antioquia y al Instituto Tecnológico Metropolitano. Adicional, se aplicó la encuesta piloto en el sector real, específicamente con algunos integrantes del área de servicio al cliente de una de las empresas de telecomunicaciones en Colombia, a la que perteneció la investigadora.

\section{Muestra y procedimientos}

El estudio se realizó en dos fases. La primera consistió en la revisión de literatura; la segunda, fue una fase descriptiva de corte transversal simple, para explicar el fenómeno de estudio por medio de ecuaciones estructurales con mínimos cuadrados parciales (PLS-SEM), y con ello medir estadísticamente las relaciones entre los constructos propuestos. A continuación, se describe la muestra seleccionada y los procedimientos correspondientes. 
En Colombia, para el primer trimestre de 2017, se tenían 17.034.012 personas con acceso a telecomunicaciones fijas (televisión: 4.081.361; internet fijo: 6.053.127; telefonía fija: 6.899.524). Las ciudades con mayor número de líneas de telefonía básica y con mayor índice de penetración de internet fijo fueron Bogotá (más de 7.000.000; 21.8\%), Medellín (más de $2.000 .000 ; 15.9 \%$ ), Cali (más de $1.500 .000 ; 14.1 \%$ ) y Barranquilla (más de $700.000 ; 12.1 \%$ ), las cuales se consideran las principales ciudades del país. Dado lo anterior, el tamaño de la muestra se definió como una no probabilística a juicio y conveniencia de la investigadora. Dicho criterio, se da por el gran tamaño de la población, que implica dificultades para abarcar toda una muestra probabilística aleatoria. Además, se requería la facilidad para segmentar la población y lograr su disponibilidad en las principales ciudades del país.

También, se eligió un cuestionario virtual para la recolección de la información, que consistió en la contratación de la empresa Código E-marketing para hacer 100 mil envíos del instrumento a las principales ciudades del país, con un segmento de personas que contaran con servicios de telecomunicaciones fijos. De los 100 mil envíos, se logró recopilar una muestra de 324 clientes (70\% pertenece al área metropolitana de Medellín; 22\% al área metropolitana de Bogotá; el resto se divide entre Cali y Barranquilla). Dicha muestra se considera significativa dada la cantidad de constructos estudiados, y por las condiciones de recolección de la información, que se dio en una época difícil del año por la temporada decembrina, vacaciones y el reintegro de los usuarios a sus puestos de trabajo.

Las personas que contestaron la encuesta son mayores de edad, entre los 25 y 49 años $(86 \%)$, solteros $(34 \%)$ y casados $(24 \%)$, empleados $(91 \%)$, con un nivel de ingresos entre MXN \$4.519 (COP \$737.717, salario mínimo en pesos colombianos para el 2017) y MXN 27.568 (COP \$4.500.000) (77\%), y con formación profesional de magísteres y doctores (83\%).

En la tabla 2, se relaciona la ficha técnica del estudio.

Tabla 2

Ficha técnica del estudio

Unidad muestral

Ámbito de estudio

Método de recogida de información

Procedimiento de muestreo

Tamaño muestral

Nivel de Confianza
Usuarios de servicios de telecomunicaciones fijos mayores de edad (> 18 años)

Medellín, Bogotá, Cali y Barranquilla y sus áreas metropolitanas

Envío de encuesta virtual

Por conveniencia y juicio

$\mathrm{n}=324$

$95 \%$ 
Error Muestral

Fecha del trabajo de campo
$5 \%$

Entre diciembre de 2016 y febrero de 2017

Fuente: Elaboración propia

Para los resultados y el análisis descriptivo, se usó el software estadístico SPSS 23. Además, se usó un Modelo de Ecuación Estructural de mínimos cuadrados parciales, para probar las hipótesis, con el software SmartPLS 3.0 (Ringle, Wende \& Becker, 2015). La elección de PLS-SEM, se debe a la propuesta de un nuevo objeto de estudio y un nuevo instrumento de medición para el sector de telecomunicaciones de Colombia (Aldás, 2017), el cual presenta bastantes elementos para medir los 4 constructos del modelo (36 ítems), y contiene dimensiones formativas y variables reflexivas (Chin, 1998; Hair et al., 2017).

\section{Presentación y Análisis de resultados}

En el modelo propuesto, el SERVQUAL contiene dimensiones formativas y se presenta como un factor de segundo orden, implicando posibles inconvenientes en el análisis PLSSEM (Aldás, 2017); es decir, el constructo calidad de servicio, se muestra como un factor no observable, sin elementos o variables asociadas, siendo los indicadores los que causan dicho factor y no al contrario. Lo anterior se resolvió con la eliminación del constructo calidad de servicio, y permitiendo que las 5 dimensiones del SERVQUAL enviaran las relaciones estructurales directamente al resto de constructos con los que tiene una relación (Hair et al., 2017; Aldás, 2017). Luego, se procedió con la estimación del modelo sin el constructo de segundo orden, cuya relación estructural se preservó manteniéndose los valores factoriales de las dimensiones y luego usándose como elementos que construyen ese constructo. De allí, se procedió con el análisis del modelo final por PLS-SEM. A continuación, se relacionan los criterios que se tuvieron en cuenta para el análisis de resultados con PLS-SEM. 
Tabla 4

Criterios para indicadores PLS-SEM

\begin{tabular}{|c|c|c|c|}
\hline $\begin{array}{l}\text { Etapas de } \\
\text { valida- } \\
\text { ción }\end{array}$ & Indicador & Criterio & Fuente \\
\hline \multirow{5}{*}{$\begin{array}{l}\text { Criterios } \\
\text { para } \\
\text { construc- } \\
\text { tos re- } \\
\text { flectivos }\end{array}$} & Alfa de Cronbach (AC) & $\Rightarrow>0.7$ & $\begin{array}{l}\text { (Nunnally \& Bernstein, } \\
\text { 1994) }\end{array}$ \\
\hline & Fiabilidad Compuesta (FC) & $\Rightarrow>0.7$ & \multirow{2}{*}{$\begin{array}{l}\text { (Fornell \& Larcker, } \\
\text { 1981) }\end{array}$} \\
\hline & $\begin{array}{l}\text { Validez convergente: media varianza } \\
\text { extraída (AVE) }\end{array}$ & $\Rightarrow>0.5$ & \\
\hline & $\begin{array}{l}\text { Validez convergente: Tamaño de las } \\
\text { cargas y nivel de significancia }\end{array}$ & $\Rightarrow>0.6 ; \mathrm{p}<0.001$ & (Bagozzi and Yi, 1988) \\
\hline & $\begin{array}{l}\text { Validez discriminante: raíces cuadradas } \\
\text { de la varianza extraída (AVE), y la rela- } \\
\text { ción Heterotrait-Monotrait (HT/MT) }\end{array}$ & $=<0.9$ & (Chin, 1998) \\
\hline \multirow{2}{*}{$\begin{array}{l}\text { Criterios } \\
\text { para } \\
\text { construc- } \\
\text { tos for- } \\
\text { mativos }\end{array}$} & $\begin{array}{l}\text { Diagnóstico de multicolinealidad: infla- } \\
\text { ción de varianza del factor (VIF) e índice } \\
\text { de tolerancia (TOL) }\end{array}$ & $\begin{array}{l}=<5(\mathrm{VIF}) \\
=>0.20(\mathrm{TOL})\end{array}$ & \multirow{2}{*}{ (Hair et al., 2017) } \\
\hline & $\begin{array}{l}\text { Análisis de peso y carga: peso significati- } \\
\text { vo, cargas, coeficientes estandarizados o } \\
\text { carga significativa. }\end{array}$ & $\mathrm{p}<0.001$ & \\
\hline \multirow{3}{*}{$\begin{array}{l}\text { Criterios } \\
\text { para el } \\
\text { Modelo } \\
\text { Estruc- } \\
\text { tural }\end{array}$} & Coeficiente de determinación (valor ). & $\begin{array}{l}=<0.75 \mathrm{y}=>0.51 \text { re- } \\
\text { levante, }=<0.50 \mathrm{y}=> \\
0.26, \text { moderado, } \mathrm{y}< \\
0.25 \text { débil }\end{array}$ & (Hair et al., 2017) \\
\hline & $\begin{array}{l}\text { Nivel de significancia de las relaciones } \\
\text { estructurales }\end{array}$ & $\mathrm{p}<0.001$ & \\
\hline & $\begin{array}{l}\text { Relevancia predictiva (): para garantizar } \\
\text { la normalidad de los datos }\end{array}$ & $>0$ & $\begin{array}{l}\text { (Stone, 1974; Geisser, } \\
\text { 1975; Tenenhaus et al., } \\
\text { 2005). }\end{array}$ \\
\hline
\end{tabular}

Fuente: Elaboración propia

La Tabla 5, muestra los promedios, desviaciones estándar (DE) y el Alfa de Cronbach de las dimensiones del modelo SERVQUAL. 
Tabla 5

Media, desviación estándar y alfa de Cronbach

\begin{tabular}{lcccc}
\hline Constructo & Media & $\begin{array}{l}\text { Desviación } \\
\text { Estándar }\end{array}$ & \multicolumn{2}{c}{$\begin{array}{l}\text { Confiabilidad Alfa de } \\
\text { Cronbach }\end{array}$} \\
\hline \hline Calidad de servicio & & & \\
Confiabilidad & 3.0 & 1.2 & 0.888 \\
Capacidad de respuesta & 2.9 & 1.1 & 0.895 \\
Seguridad & 3.3 & 1.0 & 0.894 \\
Empatía & 3.1 & 1.1 & 0.895 \\
Elementos Tangibles & 3.6 & 1.0 & 0.865 \\
\hline
\end{tabular}

Fuente: Elaboración propia

Bajo los criterios de la tabla 4, se logra confirmar con el Alfa de Cronbach, la confiabilidad de las 5 dimensiones del modelo SERVQUAL.

\section{Medición del modelo}

La Tabla 6, muestra la consistencia interna y la confiabilidad del instrumento de medida de los constructos reflexivos, analizado desde los valores aceptables del alfa de Cronbach (AC) y la Fiabilidad Compuesta (FC). También, se presentan los valores correspondientes a la validez convergente, por medio de los valores de la Varianza Extraída. Todo cumple con los criterios expuestos anteriormente, por tanto, los elementos del instrumento han medido los constructos de manera adecuada.

Tabla 6

Consistencia interna, Fiabilidad, AVE y coeficientes

\begin{tabular}{llllll}
\hline Constructos & Ítems & Cargas & AC & FC & AVE \\
& EXP1 & $* * 0.849$ & & & \\
& EXP2 & $* * 0.872$ & & & \\
Expectativas & EXP3 & $* * 0.836$ & 0.87 & 0.905 & 0.658 \\
& EXP4 & $* * 0.849$ & & & \\
& EXP5 & $* * 0.624$ & &
\end{tabular}




\begin{tabular}{|c|c|c|c|c|c|}
\hline \multirow{5}{*}{ Satisfacción } & SAT1 & $* * 0,963$ & \multirow{5}{*}{0.975} & \multirow{5}{*}{0.980} & \multirow{5}{*}{0.909} \\
\hline & SAT2 & $* * 0,959$ & & & \\
\hline & SAT3 & $* * 0,958$ & & & \\
\hline & SAT4 & $* * 0.916$ & & & \\
\hline & SAT5 & $* * 0.969$ & & & \\
\hline \multirow{5}{*}{ Valor Percibido } & VP1 & $* * 0.901$ & \multirow{5}{*}{0.96} & \multirow{5}{*}{0.97} & \multirow{5}{*}{0.877} \\
\hline & VP2 & $* * 0.937$ & & & \\
\hline & VP3 & $* * 0.956$ & & & \\
\hline & VP4 & $* * 0.948$ & & & \\
\hline & VP5 & $* * 0.941$ & & & \\
\hline
\end{tabular}

Nota: ** Indica las rutas significativas $\mathrm{p}<0.001$

Fuente: Elaboración propia. SmartPLS (Ringle, Wende \& Becker, 2015).

Para la validez discriminante, se calcularon los valores de la diagonal, correspondientes a la raíz cuadrada de la Varianza Extraída (AVE); luego, se revisó que la AVE fuera mayor que la correlación cuadrática más alta presentada debajo de la diagonal y la relación HTMT presentada en la parte superior de la diagonal. Para cada factor, se logró demostrar dicha validez (ver tabla 7).

Tabla 7

Validez Discriminante

\begin{tabular}{llll}
\hline & 1 & 2 & 3 \\
Expectativas (1) & $\underline{0.811}$ & 0.742 & 0.787 \\
Satisfacción (2) & 0.735 & $\underline{0.953}$ & 0.871 \\
Valor Percibido (3) & 0.689 & 0.847 & $\underline{0.937}$ \\
\hline
\end{tabular}

Nota: Diagonal: AVE; debajo de la diagonal: correlación al cuadrado; por encima de la diagonal: relación HTMT Fuente: Elaboración propia. SmartPLS (Ringle, Wende \& Becker, 2015).

Instrumento de medida para el constructo de segundo orden

La relevancia relativa de peso-carga de las dimensiones de la calidad de servicio (SERVQUAL), se validó mediante los pesos. Se logra evidenciar que las dimensiones son significativas ( $\mathrm{p}>0,001)$, y que las más importantes son la confiabilidad y la capacidad de respuesta (FIA: $\beta=0.382$; $C R: \beta=0.260$ ). Si bien la dimensión tangibilidad es significativa, es la que menor peso tiene ( $\mathrm{T}$ : $\beta=0.093$ ), contando por tanto con un mayor peso y una mayor carga 
las dimensiones referentes a la relación con el cliente y no la de elementos tangibles, lo cual es coherente con otros estudios (Arslan et al., 2014; Rahhal, 2015). Por otro lado, para las dimensiones formativas, se verificó la VIF, para revisar la bondad del modelo a estimar, cuyos valores deben estar por debajo de 5 con una TOL superior a 0.20 (Hair et al., 2017). Todas las dimensiones cumplieron con los criterios mencionados (ver Tabla 8).

Tabla 8

Instrumento de medida para el constructo formativo.

\begin{tabular}{|c|c|c|c|c|c|c|c|}
\hline \multirow{2}{*}{ Constructo } & \multirow{2}{*}{ Dimensiones } & \multicolumn{3}{|c|}{$\begin{array}{c}\text { Estadísticas de } \\
\text { colinealidad }\end{array}$} & \multicolumn{3}{|c|}{ Pesos-Cargas } \\
\hline & & TOL & VIF & $\begin{array}{c}\text { Beta } \\
\text { Pesos }\end{array}$ & $\begin{array}{l}\text { Sig. } \\
\text { Pesos }\end{array}$ & $\begin{array}{c}\text { Beta } \\
\text { Cargas }\end{array}$ & $\begin{array}{l}\text { Sig. } \\
\text { Cargas }\end{array}$ \\
\hline \multirow{5}{*}{$\begin{array}{l}\text { Calidad de } \\
\text { Servicio }\end{array}$} & Confiabilidad & 0.320 & 3.12 & 0.382 & 0.000 & 0.926 & 0.000 \\
\hline & Capacidad de respuesta & 0.253 & 3.96 & 0.260 & 0.000 & 0.913 & 0.000 \\
\hline & Seguridad & 0.267 & 3.74 & 0.186 & 0.000 & 0.889 & 0.000 \\
\hline & Empatía & 0.318 & 3.15 & 0.203 & 0.000 & 0.880 & 0.000 \\
\hline & Elementos tangibles & 0.513 & 1.95 & 0.093 & 0.000 & 0.701 & 0.000 \\
\hline
\end{tabular}

Fuente: Elaboración propia. SmartPLS (Ringle, Wende \& Becker, 2015).

\section{Modelo Estructural. Contraste de hipótesis}

Los resultados del contraste de las hipótesis del modelo y su capacidad predictiva, han demostrado que los valores $\mathrm{R}^{2}$ han sido estadísticamente significativos: Calidad de servicio $\left(R^{2}=0.725\right)$, Satisfacción $\left(R^{2}=0.795\right)$, y Valor percibido $\left(R^{2}=0.579\right)$. Para evaluar la relevancia predictiva $Q^{2}$ se procedió con el análisis mediante el procedimiento de re-muestreo Blindfolding, y se evidencia que todas las variables dependientes tienen relevancia predictiva: Calidad de servicio $\left(Q^{2}=0.507\right)$, Satisfacción $\left(Q^{2}=0.676\right)$, y Valor Percibido $\left(Q^{2}=0.471\right)$.

Posteriormente, se llevaron a cabo las pruebas de hipótesis calculadas por medio del Bootstrapping para obtener los coeficientes de las trayectorias y sus valores t. De las 5 hipótesis planteadas, 4 fueron significativas $(\mathrm{p}<0,001)$. Se confirman las relaciones positivas y directas de calidad de servicio - expectativas H1 ( $\mathrm{t}=53.129$, p.value $<0.05)$, calidad del servicio - satisfacción H3 ( $\mathrm{t}=6.207$, p.value $<0.05)$, calidad de servicio - valor percibido H4 ( $\mathrm{t}=34.254$, p.value $<0.05$ ), y, valor percibido - satisfacción H5 ( $\mathrm{t}=11.422$, p.value $<0.05$ ). Estos resultados van alineados con otros estudios (Zeithaml, 1988; Bolton \& Drew 1991; Spreng \& Mackoy, 1996; Ravald \& Grönroos, 1996; Taylor \& Hunter, 2003; Wang et al., 2004; Lozada \& Rodriguez, 2007; Pineda et al., 2011; Arslan et al., 2014; Bustamante, 2015; Konuk, 2018; Miranda et al., 2018; Vijayakumar \& Shivdas, 2018; Yépez et al., 2018). 
La relación expectativas y satisfacción no fue soportada $\mathrm{H} 2(\mathrm{t}=0.615$, p.value $>0.05)$. Esto se explica por la intermediación del constructo valor percibido en la relación, lo cual está alineado a los resultados de otros estudios (por ejemplo, Spreng \& Mackoy, 1996; Vigg et al., 2014; Tsafaraski et al., 2018).

Tabla 9

Resultados del modelo estructural.

\begin{tabular}{|c|c|c|c|c|c|}
\hline Hipótesis & Relaciones & Coeficientes & $\mathrm{t}$-valor & P-Valor & Conclusión \\
\hline H1 & $\mathrm{EXP} \rightarrow \mathrm{QS}$ & $* * 0.853$ & 53.129 & 0.000 & Soportada \\
\hline $\mathrm{H} 2$ & EXP $->$ SAT & 0.038 & 0.615 & 0.539 & No soportada \\
\hline $\mathrm{H} 3$ & $\mathrm{QS}->$ SAT & $* * 0.407$ & 6.207 & 0.000 & Soportada \\
\hline $\mathrm{H} 4$ & $\mathrm{QS}->\mathrm{VP}$ & $* * 0.764$ & 34.254 & 0.000 & Soportada \\
\hline H5 & VP $>$ SAT & $* * 0.510$ & 11.422 & 0.000 & Soportada \\
\hline
\end{tabular}

Nota: * *Indica las rutas significativas $\mathrm{p}<0,001$

Fuente: Elaboración propia. SmartPLS (Ringle, Wende \& Becker, 2015).

Lo anterior, por tanto, ratifica que los clientes con solo percibir un nivel de calidad del servicio alto no van a estar satisfechos (Wang et al., 2004; Vergara \& Quesada, 2011; Bustamante, 2015; Betancur et al., 2017). Es así como se aprecian las relaciones indirectas (ver tabla 10), donde un cliente satisfecho significa entregar un servicio de calidad que cumpla o supere sus expectativas y logre contemplar lo que ellos más valoran. Esto, en la misma línea de lo planteado por otros autores como Konuk (2018).

Tabla 10

Relaciones indirectas.

\begin{tabular}{lrrr}
\hline Structural path & Path Coefficient & t -value & P-Value \\
EXP $->$ QS $->$ SAT & $* * 0.347$ & 6.235 & 0.000 \\
EXP $->$ QS $->$ VP -> SAT & $* * 0.332$ & 10.747 & 0.000 \\
EXP $->$ QS -> VP & $* * 0.652$ & 25.652 & 0.000 \\
\hline
\end{tabular}

Nota: * * Indica las rutas significativas $\mathrm{p}<0,001$

Fuente: Elaboración propia. SmartPLS (Ringle, Wende \& Becker, 2015). 


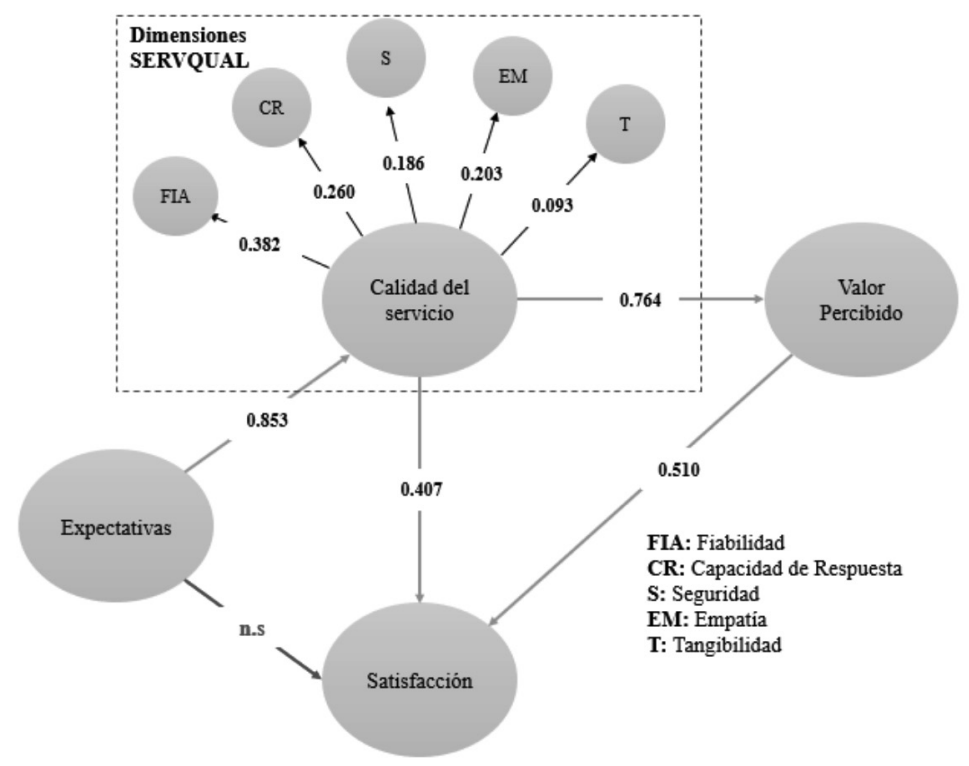

Figura. 1. Resultados del Modelo Estructural empírico del estudio con hipótesis contrastadas Fuente: Elaboración propia

\section{Conclusiones}

Esta investigación permitió mostrar los antecedentes de la satisfacción de los usuarios del servicio de telecomunicaciones fijas en Colombia, siendo relevantes los constructos propuestos, expectativas, calidad de servicio y valor percibido de manera directa e indirecta. Para un país en pleno crecimiento del sector de las telecomunicaciones y donde los estudios de este tipo son pocos (se tienen para el caso latinoamericano los de Galbán et al. 2013 y Bustamante, 2015, por ejemplo), esta investigación es un aporte a la literatura.

Además, el modelo SERVQUAL, para mostrar los determinantes de la calidad de los servicios de las empresas de telecomunicaciones en las 4 ciudades seleccionadas, resultó pertinente, y permitió validar que son más relevantes para los clientes las dimensiones de relacionamiento que la de tangibilidad (acorde con lo propuesto por Valdunciel, Flórez \& Dávila, 2007). Esto último, dado que las visitas a los puntos de atención tienden a ser esporádicas y son usadas para fines de cierre o apertura de relaciones comerciales o quejas; y si bien es importante que los puntos de atención estén bien ambientados, limpios, con equipos modernos y visiblemente atractivos, lo que realmente están evaluando de manera más positiva 
y acorde a sus expectativas, es que el servicio sea confiable y funcione bien desde el inicio, con información actual y verídica, ágil y amable, y en lo posible muy individualizada y acorde a las necesidades de los clientes.

También, se concluye que la relación expectativas y satisfacción para el caso de las telecomunicaciones en Colombia no se cumple, como en otros contextos (Bolton \& Drew, 1991; Arslan et al., 2014), dado que debe estar mediada por la misma evaluación de la calidad del servicio, y lo que valora realmente el cliente del servicio. Sin embargo, los resultados van alineados a otros estudios de sectores como el hotelero, educativo y salud (López\& Serrano, 2001; Losada \& Rodriguez, 2007; Betancur et al., 2017; Yépez et al., 2018), llevados a cabo en Colombia, lo cual contribuye a una descripción del comportamiento del consumidor colombiano sobre su valoración de la calidad de servicio y su influencia en la satisfacción. Además, esto implica que hay un reto muy grande para las empresas actuales del sector, teniendo que apostarle a la innovación en experiencia del cliente, con un buen relacionamiento y con ofertas que se adapten a lo que sus clientes más valoran y necesitan.

Por tanto, desde una perspectiva gerencial, al ser la satisfacción un factor afectivo que tiende a ser esporádico, y que requiere ser reforzado desde los mismos atributos del servicio con una suma de satisfacciones (Churchill Jr. \& Surprenant, 1982; Bolton \& Drew, 1991; Carrizo-Moreira et al., 2017), las empresas deben crear estrategias de acercamiento con los clientes, apostarle a empatizar más con ellos y tratar de dar respuestas claras, rápidas y sinceras en cada momento de verdad. Deben escucharlos en cada queja o felicitación manifiesta y propender por cumplir desde el primer momento con las promesas de servicio, establecidas de manera contractual. Además, esto debe ir acompañado con diseños de servicio flexibles, para que sus evaluaciones del servicio sean de acuerdo con lo que valoran realmente y no con la percepción de sacrificios monetarios y no monetarios.

Este estudio tiene algunas limitaciones referentes a la muestra, la cual fue seleccionada por juicio y conveniencia, aplicada solo a 4 ciudades del país, y con un instrumento específico para el sector de las telecomunicaciones en Colombia, lo cual limita la generalización de los resultados. Por lo tanto, se sugiere para investigaciones futuras utilizar muestras más amplias y replicando el instrumento en más ciudades del país, y lograr identificar escalas que permitan proponer dimensiones adicionales al caso del sector aquí estudiado, no solo las propuestas por el modelo SERVQUAL. Adicional, se sugiere revisar la percepción de los empresarios de los atributos del servicio claves para brindar un producto de calidad y con ello identificar brechas en el servicio. 


\section{Referencias}

Algarra, J. (2018). Los 5 grandes retos del sector de las telecomunicaciones. Agosto 16 de 2018. Corporación Colombia Digital. Disponible en: https://colombiadigital.net/actualidad/articulos-informativos/item/10084-los-5-grandes-retos-del-sector-de-las-telecomunicaciones.html Consultado: 22/08/2018

Aldás, J. (2017). Modelización estructural con PLS-SEM: Constructos de segundo orden. Valencia (Spain), Universitat de València-Ivie. 64p.

Arslan, M., Iftikhar M. \& Zaman R. (2014). Effect of Service Quality Dimensions on Customer Satisfaction: A Comparative Analysis of Pakistan Telecom Sector. Review of International Comparative Management. Faculty of Management, Academy of Economic Studies, Bucharest, Romania, vol. 15(4), pages 440-460, October. Recuperado de: https:// ideas.repec.org/a/rom/rmcimn/v15y2014i4p440-460 html. Consultado: 11/11/2016

Bagozzi, R. P., \& Yi, Y. (1988). On the evaluation of structural equation models. Journal of Academy of Marketing Science, 16(1), 74-94. https://doi.org/10.1007/BF02723327

Berry, L. L., Zeithaml, V. A. \& Parasuraman, A. (1985). Quality Counts in service, too. Business Horizons, p44-52. https://doi.org/10.1016/0007-6813(85)90008-4 - (1991). Understanding Customer Expectations of Service. Sloan Management Review. 32, 3. ABI/INFORM Global, pg. 39. Disponible en: https://sloanreview.mit.edu/article/understanding-customer-expectations-of-service/ Consultado: 05/06/2016

Betancur, G., D., M., Montoya, C., K. y Tavera, M., J., F. (2017). Estudio correlacional de los factores que influyen en la recomendación y la lealtad de pacientes de medicina estética Medellín Colombia, 2014. Cuadernos de Administración Journal of Management. Universidad del Valle. Vol. 33 N 58. May - August 2017. DOI: https://doi.org/10.25100/ cdea.v33i58.4527

Bolton, R. N. \& Drew, J. H. (1991). A Multistage Model of Customers' Assessments of Service Quality and Value. Journal of Consumer Research, Vol. 17, No. 4, pp. 375-384. Disponible en: https://www.jstor.org/stable/2626833. Consultado: 02/01/2017

Boohene, R. \& Agyapong, G. K.Q. (2011). Analysis of the Antecedents of Customer Loyalty of Telecommunication Industry in Ghana: The Case of Vodafone (Ghana). International Business Research, Vol. 4, No. 1. https://doi.org/10.5539/ibr.v4n1p229

Bustamante, J. C. (2015). Uso de variables mediadoras y moderadoras en la explicación de la lealtad del consumidor en ambientes de servicios. Estudios Gerenciales 3, 299-309. https://doi.org/10.1016/j.estger.2015.05.002

Carrizo-Moreira, A., Freitas-Da Silva, P., M. \& Ferreira-Moutinho, V., M (2017). The Effects of Brand Experiences on Quality, Satisfaction and Loyalty: An Empirical Study in the Telecommunications Multiple-play Service Market. Innovar [online]. 2017, vol.27, n.64, pp.23-36. ISSN 0121-5051. http://dx.doi.org/10.15446/innovar.v27n64.62366 . 
Chin, W. (1998). The partial least squares approach to structural equation modeling. Modern methods for business research. Advances in Hospitality and Leisure 8(2). Chapter 10. January 1998. Edited by G. A. Marcoulides ed. NJ: Lawrence Erlbaum Associates. 448p. ISBN 113568412X, 9781135684129. Disponible en: https://www.researchgate.net/publication/311766005_The_Partial_Least_Squares_Approach_to_Structural_Equation_ Modeling Consultado: 06/05/2016

Chiou, J. S. \& Droge, C. (2006). Service quality, trust, specific asset investment and expertise: direct and indirect effects in satisfaction-loyalty framework. Journal of the Academy of Marketing Science Volume 34, No. 4, pp 613 - 627. https://doi. org/10.1177/0092070306286934

Churchill Jr, G. A. \& Surprenant C. (1982). An investigation into the determinants of customer satisfaction. JMR, Journal of Marketing Research (pre-1986); 19, pg. 491. https:// doi.org/10.2307/3151722

Combariza, N., García, C., S., Alvarado, L., España, C. \& Rivera, H., A. (2012). Análisis estratégico del sector de Telecomunicaciones: empaquetamiento tecnológico. Bogotá: Editorial Universidad del Rosario, agosto de 2012. 36 p. (Documento de Investigación; 132) ISSN: 0124-8219. Disponible en: https://www.urosario.edu.co/urosario_files/fd/fdc423d0-4984-4cdd-a0ff-569562d16dbf.pdf. Consultado: 02/02/2017

Cronin, J., J. Jr \& Taylor, S., A. (1992). Measuring service quality: a reexamination and extensión. Journal of Marketing, Vol. 56, July, pp. 55-68. https://doi.org/10.2307/1252296

Cronin, J., J. Jr \& Taylor, S., A. (1994). SERVPERF versus SERVQUAL: Reconciling Performance-Based and Perceptions-Minus-Expectations Measurement of Service Quality. Journal of Marketing, Vol. 58, January, pp. https://doi.org/125-31. 10.2307/1252256

Escamilla-Fajardo, Paloma \& Núñez-Pomar, Juan M. (2014). Satisfacción y valor percibido en un servicio deportivo público: una propuesta de análisis y acciones de gestión. Journal of Sports Economics \& Management. 4(1). 4-21. Disponible en: https://www. researchgate.net/publication/298712051_Satisfaccion_y_valor_percibido_en_un_servicio_deportivo_publico_una_propuesta_de_analisis_y_acciones_de_gestion Consultado: $17 / 01 / 2017$

Fornell, C., \& Larcker, D. (1981). Evaluating structural equation models with unobservable variables and measurement error. Journal of Marketing Research, Vol. 18, No. 1 (Feb. 1981), pp. 39-50. https://doi.org/10.2307/3151312

Galbán, O., Clemenza, C. \& Araujo, R. (2013). Calidad de servicio en el sector de telecomunicaciones. Elemento competitivo en las empresas de televisión por suscripción. Revista Venezolana de Información, Tecnología y Conocimiento, vol. 10, núm. 2, pp. 61-82. Disponible en: http://www.redalyc.org/articulo.oa?id=82328320005 Consultado: $04 / 03 / 2017$

Geisser, S. (1975) The Predictive Sample Reuse Method with Applications, Journal of the American Statistical Association, 70:350, 320-328, https://doi.org/10.1080/01621459.1 975.10479865 
Grönroos, C. (1984). A S Service Quality Model and Its Marketing Implications. European Journal of Marketing, Vol. 18, No. 4, pp. 36-44. https://doi.org/10.1108/ EUM0000000004784

Hair, J., Hult, T., Ringle, C. \& Sarstedt, M. (2017). A primer on partial least squares structural equation modeling (PLS-SEM), Los Angeles, SAGE.

Holbrook, M. B. (1999). Introduction to consumer value. En: M. B. Holbrook (1ra Ed.), Consumer value. A framework for analysis and research, (pp. 1-28). New York: Psychology Press.

Kasiri, L. A., Cheng G., K., T., Sambasivan, M. \& Sidin, S., M. (2017). Integration of standardization and customization: Impact on service quality, customer satisfaction, and loyalty. Journal of Retailing and Consumer Services 35 (2017) 91-97. https://doi. org/10.1016/j.jretconser.2016.11.007

Kim, M. K., Park, M. C. \& Jeong, D. H. (2004). The effects of customer satisfaction and switching barrier on customer loyalty in Korean mobile telecommunication services. Telecommunications Policy 28, pp. 145-159. https://doi.org/10.1016/j.telpol.2003.12.003

Konuk, F.A. (2018). The role of store image, perceived quality, trust and perceived value in predicting consumers' purchase intentions towards organic private label food. Journal of Retailing and Consumer Services. Volume 43, July 2018, Pages 304-310. https://doi. org/10.1016/j.jretconser.2018.04.011

Lam, S., Y., Shankar, V., Erramilli, K. \& Murthy, B. (2004). Customer Value, Satisfaction, Loyalty, and Switching Costs: An Illustration From a Business-to-Business Service Context. Journal of the Academy of Marketing Science 32(3):293-311 · July 2004. https://doi. org/10.1177/0092070304263330.

López, F. M. C. \& Serrano, B. A. M. (2001). Dimensiones y medición de la Calidad de Servicio en empresas hoteleras. Revista Colombiana de Marketing, vol. 2, núm. 3. Universidad Autónoma de Bucaramanga. Disponible en: http://www.redalyc.org/articulo. oa?id=10900303 Consultado: 03/07/2017.

Losada, O., M. y Rodriguez, O., A. (2007). Calidad del servicio de salud: una revisión a la literature desde la perspectiva del marketing. Cuad. Adm. Bogotá (Colombia), 20 (34): 237-258, julio-diciembre de 2007. Disponible en: http://www.scielo.org.co/scielo.php? pid=S0120-35922007000200011\&script=sci_abstract\&tlng=es Consultado: 01/02/2018

Meesala, A. \& Paul, J. (2018). Service quality, consumer satisfaction and loyalty in hospitals: Thinking for the future. Journal of Retailing and Consumer Services 40 (2018) Pp. 261-269. https://doi.org/10.1016/j.jretconser.2016.10.011

MINTIC (2016). Boletín Trimestral de las TIC. Primer trimestre de 2016. Disponible en: http://colombiatic.mintic.gov.co/602/w3-article-15639.html

MINTIC (2017). Boletín Trimestral de las TIC. Cifras primer trimestre de 2017. Publicado: Bogotá D.C. - Colombia, julio de 2017. Disponible en: https://colombiatic.mintic.gov. co/679/articles-55212_archivo_pdf.pdf

Miranda, S., Tavares, P. \& Queiró, R. (2018). Perceived service quality and customer satisfaction: A fuzzy set QCA approach in the railway sector. Journal of Business Research 89 (2018). 371-377. https://doi.org/10.1016/j.jbusres.2017.12.040 
Naik, C., N., K., Gantasala, S., B. \& Prabhakar, G., V. (2010). SERVQUAL, Customer Satisfaction and Behavioural Intentions in Retailing. European Journal of Social Sciences. 17. Disponible en: https:/www.researchgate.net/publication/316738923_SERVQUAL_Customer_Satisfaction_and_Behavioural_Intentions_in_Retailing Consultado: 04/10/2017

Nath, P., Devlin, J. \& Reid V. (2018). The effects of online reviews on service expectations: ¿Do cultural value orientations matter? Journal of Business Research 90 (2018) 123-133. https://doi.org/10.1016/j.jbusres.2018.05.001

Naumann, E., Williams, P. \& Khan, M. S. (2009). Customer satisfaction and loyalty in B2B services: directions for future research. The Marketing Review, Vol. 9, No. 4, pp 319-333. https://doi.org/10.1362/146934709X479908

Nunnally, J. C., \& Bernstein, I. H. (1994). Psychometric Theory (3a ed.). New York: McGraw-Hill.

Oliver, R. L. (1980). A Cognitive Model of the Antecedents and Consequences of Satisfaction Decisions. JSTOR: Journal of Marketing Research, Vol. 17, No. 4, pp. 460-469. https://doi.org/10.2307/3150499

(1999a). Whence Consumer Loyalty. Journal of Marketing. Vol. 63, 3344. https://doi.org/10.2307/1252099

(1999b). Value as excellence in the consumption experience. En: M. B. Holbrook (1ra Ed.), Consumer value. A framework for analysis and research, (pp. 4362). New York: Psychology Press.

Parasuraman, A., Zeithaml, V. A. \& Berry, L. L. (1985). A Conceptual Model of Service Quality and Its Implications for Future Research. The Journal of Marketing, Vol. 49, No. 4, pp. 41- https://doi.org/50. 10.2307/1251430

Pineda, U., Estrada, M. y Parra, C., M. (2011). Aplicación del modelo Servqual y herramientas de ingeniería de la calidad para la planificación del servicio en la Biblioteca Central de la Universidad de Antioquia. Rev. Interam. Bibliot. Medellín (Colombia) Vol. $34 \mathrm{n}^{\circ}$ 32011 pp. 243-255 ISSN 0120-0976. Disponible en: http://www.scielo.org.co/pdf/rib/ v34n3/v34n3a1.pdf Consultado: 03/02/2017

Rahhal, W. (2015). The Effects of Service Quality Dimensions on Customer Satisfaction: An Empirical Investigation in Syrian Mobile Telecommunication Services. International Journal of Business and Management Invention, ISSN (Online): 2319 - 8028, ISSN (Print): 2319 - 801X. Volume 4 Issue 5. May. 2015, PP-81-89. Disponible en: https:// www.ijbmi.org/papers/Vol(4)5/L045081089.pdf Consultado: 06/02/2017

Ravald, A. \& Grönroos, C. (1996). The value concept and relationship. European Journal of Marketing. 30(2):19-30 · February 1996. https://doi.org/10.1108/03090569610106626

El Tiempo (2015). Más de 3,5 millones de colombianos han cambiado de operador móvil. El Tiempo. Redacción Tecnósfera, 2015. Disponible en: http://www.eltiempo.com/archivo/ documento/CMS-15258126

Ringle, C. M., Wende, S., and Becker, J.-M. 2015. "SmartPLS 3.” Boenningstedt: SmartPLS $\mathrm{GmbH}$, http://www.smartpls.com 
Roca, C., A., Cabello, M., D., Gonzalez, J. y Courel, I., J. (2018). Estudio de satisfacción de los asistentes a la Universiada de Invierno Granada. Retos, número 33, $2018\left(1^{\circ}\right.$ semestre). ISSN: Edición impresa: 1579-1726. Edición Web: 1988-2041. Disponible en: https://dialnet.unirioja.es/servlet/articulo?codigo=6367768 Consultado: 04/05/2018

Sirdeshmukh, D., Jagdip S. \& Sabol, B. (2002). Consumer trust, value, and loyalty in relationship exchanges. Journal of marketing. 66 (january). 15-37. https://doi.org/10.1509/ jmkg.66.1.15.18449

Spreng, R. A. \& Mackoy, R. D. (1996). An Empirical Examination of a Model of Perceived Service Quality and Satisfaction. Journal of Retailing, Volume 72(2), pp. 201-214. https://doi.org/10.1016/S0022-4359(96)90014-7

Stone, M. (1974). Cross-Validatory Choice and Assessment of Statistical Predictions. Journal of the Royal Statistical Society. Series B (Methodological), 36(2), 111-147. Retrieved May 15, 2020. Disponible en www.jstor.org/stable/2984809 Consultado 06/06/2016

Strenitzerová, M. \& Gaňa, J. (2018). Customer Satisfaction and Loyalty as a Part of Customer-Based Corporate Sustainability in the Sector of Mobile Communications Services. Sustainability 10(5):1657. May 2018. https://doi.org/10.3390/su10051657

Taylor, S. A. \& Hunter, G. (2003). An exploratory investigation into the antecedents of satisfaction brand attitude and loyalty between the (B2B) eCRM industry. Journal of Consumer Satisfaction, Dissatisfaction and Complaining Behavior. Vol. 15 pp 19-35. Disponible en: http://search.ebscohost.com.itm.elogim.com/login.aspx?direct=true\&db=bth\&AN=101101006\&lang=es\&site=ehost-live Consultado: 02/03/2017

Tenenhaus, M., Esposito Vinzi, V., Chatelin, Y., and Lauro, C. (2005). PLS path modeling. Computational Statistics \& Data Analysis, Volume 48, Issue 1, 1 January 2005, Pages 159-205. https://doi.org/10.1016/j.csda.2004.03.005

Tsafaraski, S., Kokotas, T. \& Pantouvakis, A. (2018). A multiple criteria approach for airline passenger satisfaction measurement and service quality improvement. Journal of air transport management (68), 2018, 61-75. https://doi.org/10.1016/j.jairtraman.2017.09.010

Umar, A. \& Bahrun, R. (2017). The mediating relationship of customer satisfaction between brand trust, brand social responsibility image with moderating role of switching cost. Advanced Science Letters. Volume 23, Issue 9, September 2017, Pages 9020-9025. https:// doi.org/10.1166/asl.2017.10015

Valdunciel B. L., Flórez, R. M. y Miguel, D. J. A. (2007). Análisis de la calidad del servicio que prestan las entidades bancarias y su repercusión en la satisfacción del cliente y la lealtad hacia la entidad. Revista Asturiana de Economía - RAE No. 38, pp. 79-107. Disponible en: https://dialnet.unirioja.es/servlet/articulo?codigo=3017921 Consultado: 03/07/2020

Vergara, S., J., C. y Quesada, I., V., M. (2011). Análisis de la calidad en el servicio y satisfacción de los estudiantes de Ciencias Económicas de la Universidad de Cartagena mediante un modelo de ecuaciones estructurales. Revista Electrónica de Investigación Educativa, 13(1), 108-122. Disponible en: http://redie.uabc.mx/vol13no1/contenido-vergaraquesada.html Consultado: 03/02/2017 
Vergara, J. C., Quesada, V. M. y Blanco, I. (2011). Análisis de la calidad en el servicio y satisfacción de los usuarios en dos hoteles cinco estrellas de la ciudad de Cartagena (Colombia) mediante un modelo de ecuaciones estructurales. Ingeniare Revista chilena de ingeniería, vol. 19 N 3 , pp. 420-428. http://dx.doi.org/10.4067/S0718-33052011000300011

Vigg, K. S. \& Bhargav, A. (2014). Service Quality Expectations and Perceptions of Telecom Sector in India. International Journal of Advancements in Technology, Vol. 5 No. 1. Disponible en: https://pdfs .semanticscholar.org/72f4/b6c7763d3639d33bf80be905711b01a74ca1.pdf Consultado: 02/01/2017

Vijayakumar, V. \& Shivdas, A. (2018). Understanding drivers of customer satisfaction in Indian telecom sector: A PLS-SEM based approach. International Journal of Pure and Applied Mathematics Open Access. Volume 119, Issue Special Issue 7A, 2018, Pages 253-260. Disponible en: https://acadpubl.eu/jsi/2018-119-7/articles/7a/28.pdf Consultado: 04/06/2018

Wang, Y., Lo, H. P. \& Yang, Y. (2004). An Integrated Framework for Service Quality, Customer Value, and Satisfaction: Evidence from China's Telecommunication Industry. Information Systems Frontiers 6:4, 325-340, 2004. https://doi.org/10.1023/B:ISFI.0000046375.72726.67

Yépez, C., M., C., Ricaurte, C., M., y Jurado, F., D., M. (2018). Calidad percibida de la atención en salud en una red pública del municipio de Pasto, Colombia. Univ. Salud. 2018;20(2):97-110. DOI: http://dx.doi.org/10.22267/rus.182002.114

Zeithaml, V. A. (1981). How consumer evaluation differ between goods and services. Texas A\&M University. Pp 186 - 190. Disponible en: https:/www.researchgate.net/publication/279912843_How_Consumer_Evaluation_Processes_Differ_for_Products_and_ Services

(1988). Consumer Perceptions of Price, Quality, and Value: A MeansEnd Model and Synthesis of Evidence. Journal of Marketing Vol. 52, 2-22. https://doi. org/10.2307/1251446

Zeithaml, V. A., Berry, L. L. \& Parasuraman, A. (1993). The Nature and Determinants of customer Expectations of service. Journal of the Academy of Marketing Science. Vol. 21, \#1, pp. 1-12. https://doi.org/10.1177/0092070393211001

(1996). The Behavioral Consequences of Service Quality. Journal of Marketing, Vol. 60, No. 2, pp. 31-46. https://doi.org/10.2307/1251929

Zeithaml, V. A., Bitner, M. J. y Gremler, D. D. (5ta Ed.) (2009). Marketing de servicios. McGraw-Hill Interamericana de España S.L. 740 p.

Zhang, Y-L., Bi, R-F, \& Xiao, M. (2017). Studying on Enhancing Readers' Satisfaction Model of Electronic Service Quality in Library Based on LibQUAL+ and Kano. Procedia Engineering 174 (2017) 260 - 266 13th Global Congress on Manufacturing and Management, GCMM 2016. https://doi.org/10.1016/j.proeng.2017.01.132 